\title{
Universiteit
}

Leiden

The Netherlands

\section{Charge carrier localization due to ferromagnetic clusters in concentrated AuFe alloys}

Gorshunov, B.; Kaiser, S.; Zhukova, E.S.; Prokhorov, A.S.; Hesselberth, M.B.S.; Aarts, J.; ... ; Dressel, M.

\section{Citation}

Gorshunov, B., Kaiser, S., Zhukova, E. S., Prokhorov, A. S., Hesselberth, M. B. S., Aarts, J., ... Dressel, M. (2009). Charge carrier localization due to ferromagnetic clusters in concentrated AuFe alloys. Physical Review B, 79(5), 054203. doi:10.1103/PhysRevB.79.054203

Version: $\quad$ Not Applicable (or Unknown)

License: $\quad$ Leiden University Non-exclusive license

Downloaded from: https://hdl.handle.net/1887/45146

Note: To cite this publication please use the final published version (if applicable). 


\title{
gु \\ Charge carrier localization due to ferromagnetic clusters in concentrated AuFe alloys
}

\author{
B. Gorshunov, ${ }^{1,2}$ S. Kaiser, ${ }^{1}$ E. S. Zhukova, ${ }^{2}$ A. S. Prokhorov, ${ }^{2}$ M. B. S. Hesselberth, ${ }^{3}$ J. Aarts, ${ }^{3}$ \\ G. J. Nieuwenhuys, ${ }^{3}$ and M. Dressel ${ }^{1}$ \\ 1. Physikalisches Institut, Universität Stuttgart, Pfaffenwaldring 57, 70550 Stuttgart, Germany \\ ${ }^{2}$ A.M. Prokhorov General Physics Institute, Russian Academy of Sciences, Moscow, Russia \\ ${ }^{3}$ Kamerlingh Onnes Laboratory, Leiden University, Leiden, The Netherlands
}

(Received 2 December 2008; revised manuscript received 21 January 2009; published 18 February 2009)

\begin{abstract}
The electrodynamic properties of $\mathrm{Au}_{1-x} \mathrm{Fe}_{x}$ films are measured for various Fe contents $x=0,6,17$, and 22 at. \%. From room-temperature optical measurements over a broad spectral range $\left(10-3.3 \times 10^{4} \mathrm{~cm}^{-1}\right)$, we obtain the frequency-dependent conductivity of the films. As the iron concentration increases, a stronger decrease in the magnetic contribution to the dc conductivity compared to ac conductivity (at frequency $10 \mathrm{~cm}^{-1}$ ) is detected. In addition, a broad absorption peak is discovered at frequency $1000-3000 \mathrm{~cm}^{-1}$ for concentrations of 6-22 at. \%. Both observations are associated with ferromagnetic clusters that cause partial localization of conduction electrons.
\end{abstract}

DOI: 10.1103/PhysRevB.79.054203

PACS number(s): 75.50.Lk, 71.55.Jv, 72.15.Rn

\section{INTRODUCTION}

In spite of a long history of intense theoretical and experimental research, there are still quite a number of unresolved problems concerning the nature and the properties of the spin-glass state. ${ }^{1,2}$ Some of these problems concern the nature of the phase transition to the glassy state; for instance whether this transition can be considered as a thermodynamical phase transition since the spin-glass phase is completely disordered. Other properties are also not completely understood yet, such as the magnetic memory and hysteresis effects, the mechanisms of magnetic interactions in systems with different concentrations of magnetic component, etc. These open questions put spin glasses among the most interesting topics of modern solid-state physics and call for further experimental and theoretical research.

Since delocalized electrons play a fundamental role in mediating the Ruderman-Kittel-Kasuya-Yosida (RKKY) interaction between the magnetic inclusions and in forming the spin-glass state, the investigation of their properties is of basic importance. A direct way to do so is provided by measuring the electrical resistivity as a function of temperature, $\rho(T)$. In a series of thorough experiments on the most comprehensively studied material, AuFe, ${ }^{3-7}$ the rather intricate behavior of $\rho(T)$ is observed which reflects the complexity of the spin-glass phenomenon. At the lowest temperatures (around $1-2 \mathrm{~K}$ ), the magnetic part of the resistivity $\rho_{\text {magn }}$ (i.e., the difference between the measured resistivity and the resistivity $\rho_{\mathrm{Au}}$ of the host, pure Au) obeys the dependence $\rho_{\text {magn }}(T) \propto T^{3 / 2}$. Upon heating and approaching the transition (freezing) temperature $T_{f}$ the dependence transforms into the linear one, $\rho_{\text {magn }}(T) \propto T$. At even higher temperatures a decrease in $\rho_{\text {magn }}$ is seen leading to a broad maximum. The resistivity is strongly dependent on concentration $x$ of the magnetic component. Three correspondent regimes can be distinguished in AuFe. At low concentrations, $x<0.5$ at. \% of iron, the Kondo-type scattering off of independent moments prevails at low temperatures. Around $8-10$ at. \% Fe, the Fe magnetic moments experience the RKKY interaction and the spin-glassy state emerges. In alloys with higher con- centration of up to approximately 15 at. \%, complicated electronic and magnetic behaviors are observed which, in addition, are connected with the formation of ferromagnetic iron clusters. In this range of concentrations, electrons are believed to scatter off both clusters and those unordered $\mathrm{Fe}$ moments which do not belong to the clusters. For even higher concentrations (above 15-18 at. \%), the properties of the material are determined by a complicated interplay of the RKKY interaction between isolated $\mathrm{Fe}$ moments and the direct-exchange intercluster and interionic interactions. It is assumed that the maximum of $\rho_{\text {magn }}(T)$ appears due to the competition between the low-temperature scattering of electrons located within the matrix of unordered frozen impurity magnetic moments, on one hand, and high-temperature modified Kondo-type scattering off clusters, on the other hand. However, there is still no microscopic theory or model which could reproduce the observed $\rho_{\text {magn }}(T, x)$ behaviors and provide more insight into the physics of spin glass and cluster states in AuFe and other representatives of this material class.

Optical spectroscopy is the most suitable tool to study the dynamics of charge carriers for it gives complementary information on their microscopic characteristics such as mobility, concentration, scattering rate, plasma frequency, etc. ${ }^{8} \mathrm{Re}-$ cently we have applied optical spectroscopy for the first time to investigate spin glasses. ${ }^{9}$ At room temperature an increase in the carrier scattering rate with growing $x$ was found to be caused by electron collisions with magnetic moments of $\mathrm{Fe}$. In the spin-glass phase, at $T=5 \mathrm{~K}$, a pseudogap in the conductivity spectrum for the $x=6$ at. \% composition was detected with the magnitude close to the RKKY energy for $\mathrm{AuFe}, \Delta_{\mathrm{RKKY}} \approx 2.2 \mathrm{meV}$, and the origin of the pseudogap was associated with partial localization of electrons which mediate the RKKY interaction between localized Fe centers.

Here we present results of our optical investigation of $\mathrm{AuFe}$ alloys with significantly higher concentrations of iron in the gold matrix. Our aim is to study the influence of cluster effects on the electronic properties of AuFe and to investigate the crossover from spin-glass state to a dilute ferromagnetic or macroscopically ordered state. 


\section{EXPERIMENTAL DETAILS}

To prepare the thin films of AuFe used in this study, the high-purity metals were cosputtered onto high-resistive crystalline silicon substrates (of size $10 \times 10 \mathrm{~mm}^{2}$ and thickness of about $0.5 \mathrm{~mm}$ ). Before the argon sputter gas was admitted, the equipment was pumped down to UHV conditions of a pressure of $10^{-7}$ Torr to prevent oxidation of the films during fabrication. The films were analyzed using Rutherford backscattering and electron microprobe analysis; the film thicknesses (approximately $35 \mathrm{~nm}$ ) and compositions were found to be homogeneous. Measurements of the magnetic susceptibility and relaxation of low-energy muons performed on films of the same batch and with lower Fe content reliably indicate the transition to the spin-glass state (see Ref. 10 and references therein) with the freezing temperatures $T_{f}$ agreeing with those obtained for bulk AuFe samples.

For the optical measurements a set of different spectrometers was employed. At the low-frequency end, between 10 and $30 \mathrm{~cm}^{-1}$, a quasioptical spectrometer was used based on monochromatic and frequency tunable sources of radiationbackward-wave oscillators. ${ }^{11}$ During the measurement the spectrum of transmission coefficient of the film on transparent silicon substrate is recorded. With the dielectric parameters of the substrate determined beforehand, the conductivity of the films is determined by fitting the transmissivity spectra with the corresponding expressions for the two-layer system. $^{8,12}$ In the infrared spectral range $\left(200-8000 \mathrm{~cm}^{-1}\right)$, reflectivity spectra $R(\nu)$ were measured using an infrared microscope connected to a Bruker IFS 66v Fourier-transform spectrometer. An aluminum mirror served as reference; its reflectivity was corrected by the literature data. ${ }^{13} \mathrm{~A}$ Woollam variable-angle spectroscopic ellipsometer equipped with a Berek compensator was utilized to measure in the frequency range between 5000 and $3.3 \times 10^{4} \mathrm{~cm}^{-1}$ with a resolution of $200 \mathrm{~cm}^{-1}$ under multiple angles of incidence between $65^{\circ}$ and $85^{\circ}$. From the ellipsometric measurements the real and imaginary parts of the refractive index are obtained which then allow us to directly evaluate any optical parameter such as the reflectivity $R(\nu)$ or the conductivity $\sigma(\nu)$. All measurements were performed at room temperature.

\section{EXPERIMENTAL RESULTS}

Figure 1 shows broadband spectra of the reflection coefficient of (a) a pure gold film and (b)-(d) three $\mathrm{Au}_{1-x} \mathrm{Fe}_{x}$ films on Si substrates with concentrations of Fe $x=6,17$, and 22 at. \%, respectively. The data of frames (a) and (b) have been published previously. ${ }^{9}$ While in the infrared frequency range the reflectivity $R(\nu)$ is measured directly, the terahertz reflectivity is calculated on the basis of the conductivity determined from the transmission coefficient spectra. In the near infrared to ultraviolet range, $R(\nu)$ is also calculated from ellipsometric directly measured optical parameters of the films. The oscillations observed at the low-frequency end come from multireflections within the plane-parallel Si substrates.

All spectra shown in Fig. 1 are typical for a metal; the reflectivity is high at low frequencies, and there is a clear plasma edge at the high-frequency end, around 2

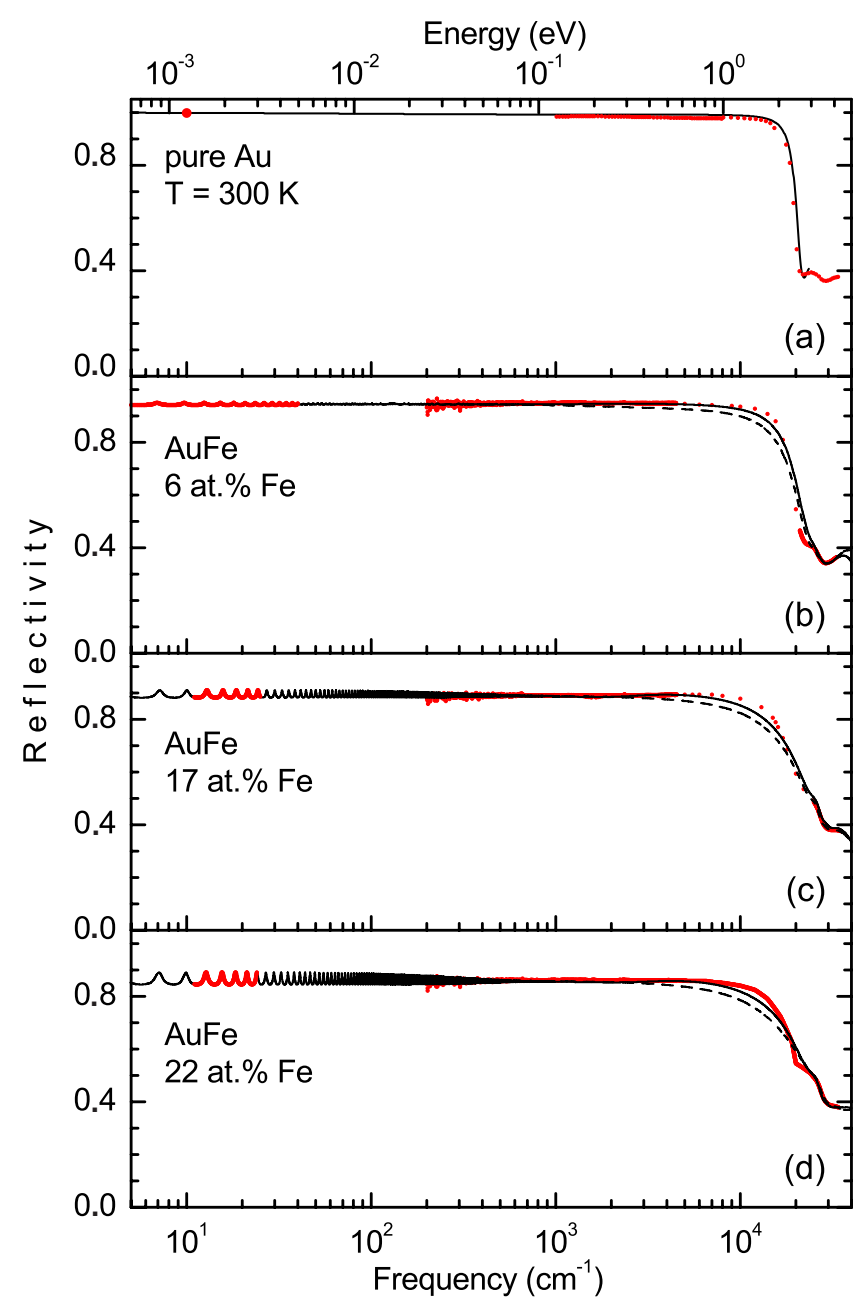

FIG. 1. (Color online) Reflectivity spectra of four films on a silicon substrate: (a) pure gold (thickness of $50 \mathrm{~nm}$ ) and (b)-(d) $\mathrm{Au}_{1-x} \mathrm{Fe}_{x}$ (thickness of $35 \mathrm{~nm}$ ) with various $\mathrm{Fe}$ concentrations $x$. The dashed lines represent a fit by the Drude expression (1) for the conductivity of the film. The solid lines correspond to a fit including Drude (1) and Lorentzian (2) terms. Multireflections within the substrate cause the interference fringes at low frequencies.

$\times 10^{4} \mathrm{~cm}^{-1}$; at even higher frequencies electronic interband transitions are seen. With increasing Fe content, the absolute value of the low-frequency reflectivity drops and the plasma edge gets smoother. The decrease in the reflectivity for higher Fe concentrations is connected with a decrease in the $\mathrm{dc}$ and low-frequency conductivity, as discussed below in the following paragraph. The blackdashed and solid lines in Fig. 1 present fits of the reflectivity spectra assuming a twolayered system (film on a substrate). The dashed lines correspond to cases when only a Drude term for the complex conductivity was used to model the response of free carriers in the film, ${ }^{8}$

$$
\sigma_{D}(\nu)=\frac{\sigma_{0}}{1-i \nu / \gamma_{D}},
$$

where $\sigma_{0}=\sigma(\nu \rightarrow 0)$ is the zero-frequency conductivity and $\gamma_{D}$ is the relaxation rate of charge carriers. The interband transitions seen at the highest frequencies (above $10^{4} \mathrm{~cm}^{-1}$ ) 
were described with a Lorentzian oscillator term, ${ }^{8}$

$$
\sigma_{L}(\nu)=\frac{0.5 \Delta \epsilon \nu_{0}^{2} \nu}{\nu \gamma_{L}+i\left(\nu_{0}^{2}-\nu^{2}\right)},
$$

where $\Delta \epsilon$ is the dielectric contribution (strength) of the oscillator, $\nu_{0}$ is the resonance frequency, and $\gamma_{L}$ is the damping. The reflectivity spectrum of pure gold film below the plasma edge is well described by the Drude reflectivity based on Eq. (1). For all AuFe films, however, clear deviations are observed at frequencies around $10^{4} \mathrm{~cm}^{-1}$, close to the position of the plasma edge. They indicate the presence of an additional band in this frequency range. We modeled this band with the Lorentzian term (2). As will be described later, the center frequency of the band shifts from roughly 1000 to $3000 \mathrm{~cm}^{-1}$, and the damping increases from 2000 to $5000 \mathrm{~cm}^{-1}$ with $\mathrm{Fe}$ content. The solid lines in Fig. 1 show the results of fits, when both terms (1) and (2) were used. For all three films the quality of the fit around $10^{4} \mathrm{~cm}^{-1}$ could be further improved by adding more Lorentzians; this implies that the shape of the band is more complicated than a simple Lorentzian.

The conductivity spectra of the five films are displayed in Fig. 2. The dashed lines show the fits using just a Drude term (with Lorentzians for the interband transitions). The solid lines are fits with both taken into account, a Drude term [Eq. (1)] and a Lorentzian oscillator [Eq. (2)]. The Lorentzian contributions are indicated separately by the dotted lines. As best seen from the compilation in panel (e), the lowfrequency conductivity is reduced and the charge carrier scattering rate becomes larger as the iron concentration increases. These behaviors are given in details in Fig. 3, together with the concentration dependence of other Drude and Lorentz term parameters. The decrease in the plasma frequency of free carriers when going from pure gold to AuFe is a consequence of the spectral weight transfer from the Drude term to the Lorentzian. The analysis of the conductivity spectra reveals that the loss of the Drude spectral weight,

$$
f_{D}=\nu_{p}^{2} / 8=\frac{n e^{2}}{8 \pi m}
$$

is recovered by the spectral weight of the Lorentz oscillator $f_{L}=\Delta \epsilon \nu_{0}^{2} / 8 . \quad\left(\nu_{p}\right.$ is the plasma frequency of the free charge carriers; $n, e$, and $m$ are their concentration, charge, and mass, respectively.) The lower panel of Fig. 3 presents the concentration dependences of the magnetic contribution to the resistivity by choosing the ac conductivity at a frequency of $10 \mathrm{~cm}^{-1}$ and taking the dc data from Ref. 14. The magnetic contribution is obtained by subtracting the resistivity of pure gold $\rho_{\mathrm{Au}}$ from the total resistivity. For low Fe concentrations $(x \leq 4$ at. \%) the dc and the ac values practically coincide, while for larger iron content the dc magnetic resistivity is significantly higher. This is also demonstrated by the inset of Fig. 2(a) where the ac $\left(10 \mathrm{~cm}^{-1}\right)$ and the dc conductivities are presented for different $\mathrm{Fe}$ concentrations; the higher the Fe concentration, the larger is the ac conductivity compared to the dc one.

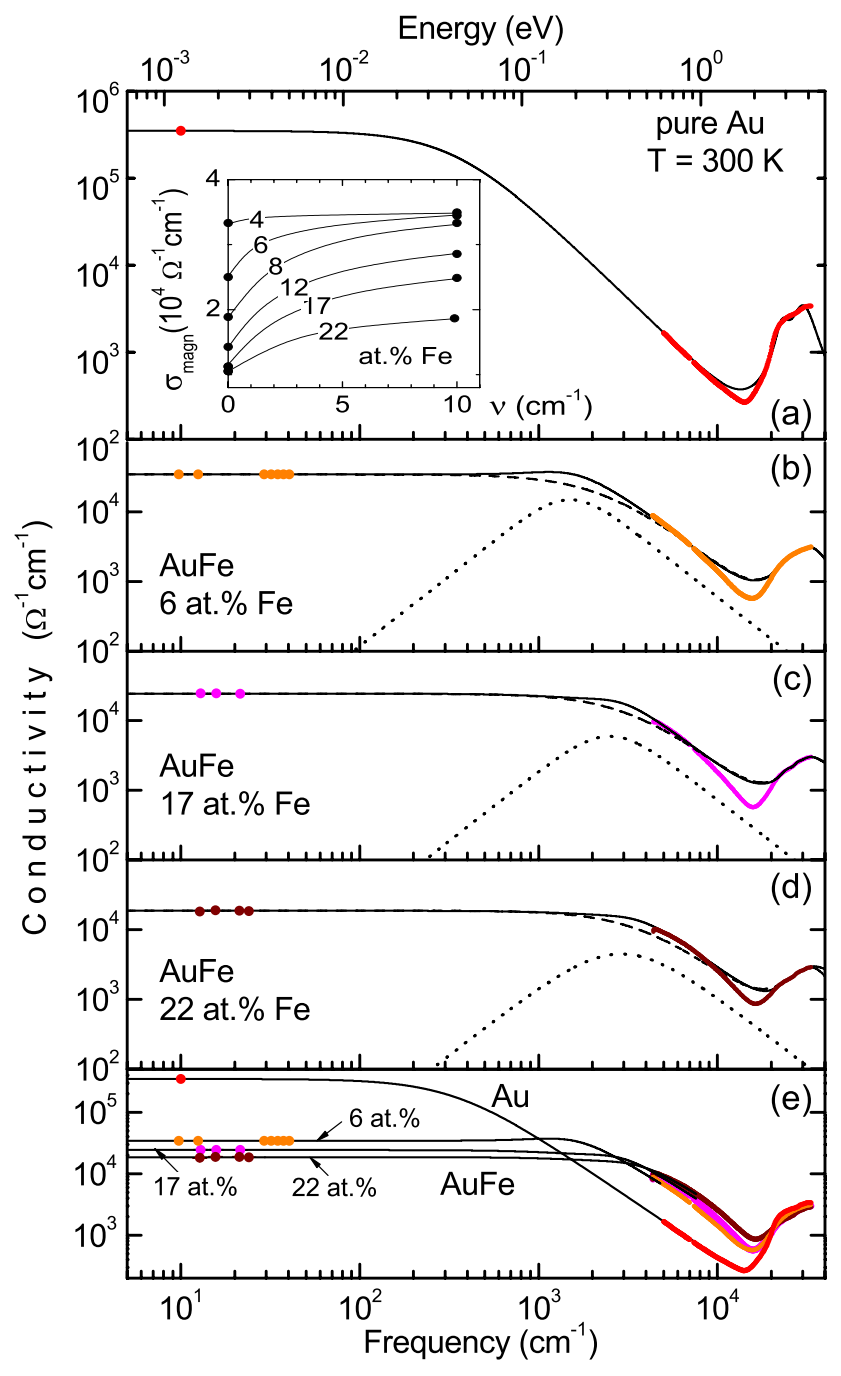

FIG. 2. (Color online) Optical conductivity of (a) pure gold and (b)-(d) of AuFe films with various Fe concentrations plotted over a wide frequency range. The colored dots are the results of direct measurements. The dashed and solid lines correspond to fits of the reflectivity spectra (Fig. 1) using either a Drude term [Eq. (1)] or a combination of a Drude and a Lorentzian (2) term, respectively. The dotted lines show the Lorentzian contribution separately, which moves to higher frequencies as the iron content increases. Panel (e) summarizes the results for the different films. The inset shows the ac $\left(10 \mathrm{~cm}^{-1}\right)$ and the dc (Ref. 14) magnetic conductivities of AuFe for different Fe concentrations; lines are guides to the eye.

\section{DISCUSSION}

In pure gold, phonons are the main scattering mechanism at high temperatures. When iron is added to the gold matrix, we observe an increase in the free-carrier scattering rate $\gamma_{D}$ by about a factor of 10 for $x=22$ at. $\%$. We ascribe this change in the scattering mechanism to the additional magnetic scattering in AuFe. The rise of $\gamma_{D}$ and the slight decrease in the carrier plasma frequency lead to a reduction in the low-frequency $\left(\nu \ll \gamma_{D}\right)$ conductivity.

The magnetic contribution to the zero-frequency resistivity can be calculated via $1 / \sigma_{0}-\rho_{\mathrm{Au}}$, where $\sigma_{0}$ is obtained from the fit of the reflectivity spectra by the Drude expres- 


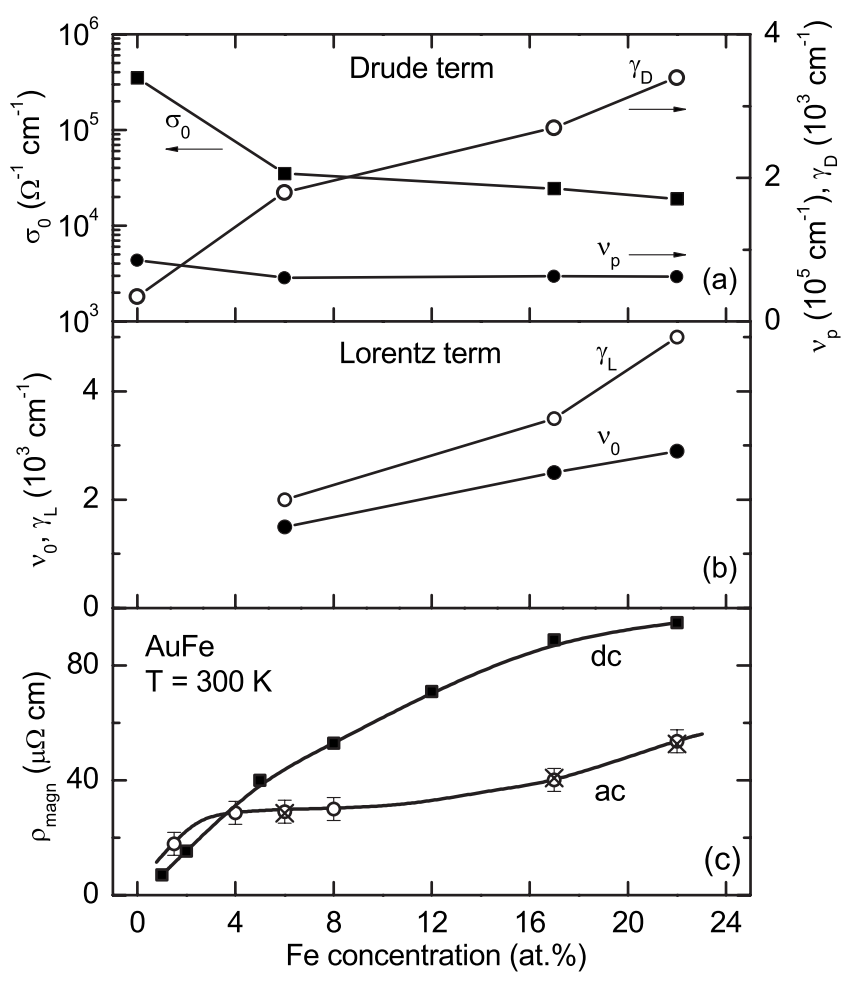

FIG. 3. Dependence of the optical and transport parameters of $\mathrm{Au}_{1-x} \mathrm{Fe}_{x}$ films on Fe concentration $x$. (a) Zero-frequency conductivity $\sigma_{0}$ (left axis), plasma frequency $\nu_{p}$, and scattering rate $\gamma_{D}$ of the Drude term [Eq. (1)] (right axis). (b) Eigenfrequency $\nu_{0}$ and damping $\gamma_{L}$ of the Lorentz term [Eq. (2)]. (c) Dependences of the magnetic contributions to the resistivity on the iron concentration. The dc data (squares) are taken from Ref. 14. For the ac data, the open circles are obtained from direct conductivity measurements at terahertz frequencies with the resistivity of gold subtracted, $1 / \sigma_{\mathrm{ac}}$ $-\rho_{\mathrm{Au}}$. The crosses correspond to the difference of the $\nu \rightarrow 0$ conductivity $\sigma_{0}$, as obtained from fits of the reflectivity by the Drude model over a large frequency range, compared to the resistivity of gold, $1 / \sigma_{0}-\rho_{\mathrm{Au}}$.

sion (1). Its concentration dependence is presented by crosses in Fig. 3(c) together with the dependence of the ac magnetic resistivity $\rho_{\mathrm{ac}}^{\mathrm{magn}}=1 / \sigma_{\mathrm{ac}}-\rho_{\mathrm{Au}}$ directly measured at $10 \mathrm{~cm}^{-1}$ (circles). Both quantities practically coincide, as expected for $\nu \ll \gamma_{D}$ in case the Drude model is applicable. At the same time the concentration dependence of the ac magnetic resistivity is significantly different from that of the dc magnetic resistivity $\rho_{\mathrm{dc}}^{\text {magn }}$ obtained by direct dc measurements on bulk samples. ${ }^{14}$ While for low iron content $x$ $\leq 4$ at. $\%$, the two quantities $\rho_{\mathrm{dc}}^{\mathrm{magn}}$ and $\rho_{\mathrm{ac}}^{\mathrm{magn}}$ are equal within the experimental uncertainty, significant deviations are found for large $x$; the ac component is much smaller than the dc property, $\rho_{\mathrm{ac}}^{\text {magn }}<\rho_{\mathrm{dc}}^{\text {magn }}$.

Based on numerous experiments over the last decades, the idea was established that due to direct-exchange coupling between the $\mathrm{Fe}$ moments, ferromagnetic clusters form and influence the physical properties and the phase diagram of AuFe. ${ }^{15-19}$ In a rather broad range of temperatures and $\mathrm{Fe}$ concentrations, the properties of this system are governed by an interplay of RKKY and direct-exchange interactions between the Fe moments. ${ }^{4,5,14,20}$ The role that the clusters play

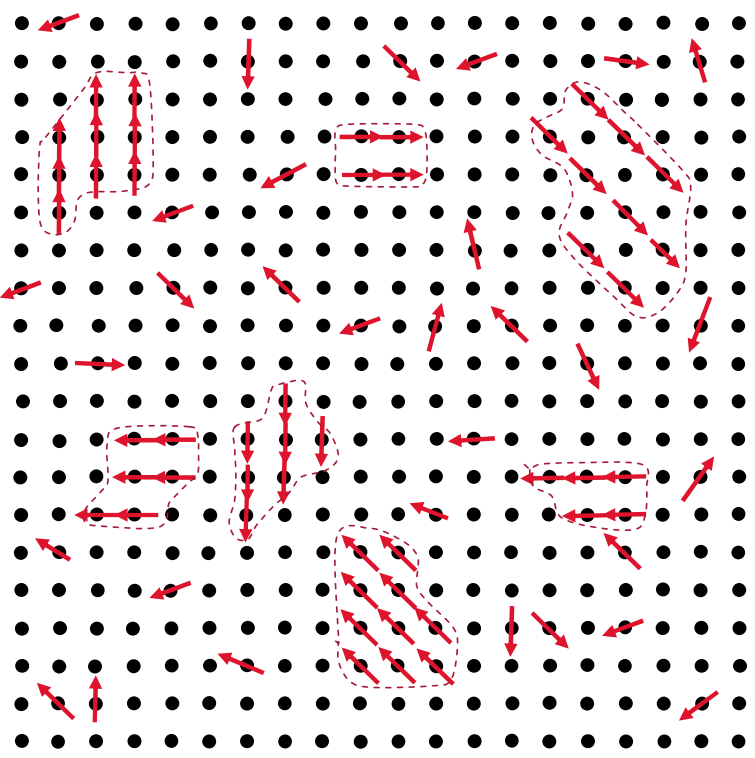

FIG. 4. (Color online) Sketch of the magnetic structure of AuFe alloy with iron concentration of 20 at. \%. Dots represent $\mathrm{Au}$ and dots with red arrows (spins) represent Fe. Ferromagnetic clusters of oriented Fe spins are indicated by the dashed lines. The figure is drawn following the suggestion of Ref. 22.

becomes most important at high concentrations, above some critical value of $x=12-15$ at. $\%$. However, already at rather low values of $x$, even in the spin-glass phase, clusters affect the behavior of AuFe alloys. ${ }^{4,21}$ On the other hand, the RKKY interaction which is dominant in the spin-glass state also influences the properties for concentrations as high as $x=17$ at. $\% .^{16,20}$

Our experimental findings suggest that ferromagnetic clusters exist in concentrated AuFe already at room temperature and that they are responsible for the observed difference between the magnetic components of the dc and ac resistivities as well as for the excitations discovered at 1000-3000 $\mathrm{cm}^{-1}$ (Fig. 2). For low concentrations, the magnetic moments are statistically distributed and randomly oriented. As $x$ increases the moments are grouped in some regions forming magnetically ordered clusters. In a simplified picture, $^{22}$ the magnetic structure of clustered AuFe is sketched in Fig. 4, corresponding to a concentration of 20 at. $\%$ of iron.

Within the clusters the conduction electrons interact with the ordered Fe moments, which decreases their energy by an amount given by the exchange coupling between the electron spin and the $\mathrm{Fe}$ moments and which leads to less magnetic scattering compared to the scattering off of randomly oriented moments in the $\mathrm{Au}$ matrix. In addition, the electrons experience the impeding potential from magnetic inhomogeneities at the cluster boundary. This can lead to an effective localization of conduction electrons within the clusterssome sort of magnetic polaronic effect-and cause excess resistance in the bulk material. Similar effects are observed in conducting magnetic systems with domain walls or grain boundaries. ${ }^{23,24}$ In general, localization causes a frequencydependent low-frequency conductivity that follows a power law $\sigma_{\text {ac }}(\nu) \propto \nu^{s}$ with $s \approx 1$ (Ref. 25); correspondingly, the ac 
resistivity is $\rho_{\mathrm{ac}} \propto \nu^{-s}$, implying that $\rho_{\mathrm{dc}}>\rho_{\mathrm{ac}}$, as observed in our AuFe samples. From the inset of Fig. 2 it is seen that the dispersion $\sigma_{\mathrm{ac}}(\nu) \propto \nu^{s}$ takes place below the lowest frequencies of our measurements $\left(10 \mathrm{~cm}^{-1}, 300 \mathrm{GHz}\right)$ and starts to develop for $x>4$ at. $\%$.

In addition to the reduction in conductivity with rising $\mathrm{Fe}$ content in AuFe, we observe a broad absorption band at around $1000-3000 \mathrm{~cm}^{-1}$. The spectral weight of the band is transferred from the itinerant electrons in pure Au to the peak in AuFe, which indicates its purely electronic character. We ascribe it to the electrons confined in the magnetic clusters. Compared to the gold matrix with disordered iron atoms, the clusters of ordered magnetic moments provide an advantageous environment in which the conduction electrons experience scattering events with a reduced rate. ${ }^{26}$ The partial localization of the electrons may lead to a resonancelike band which in a first approximation can be modeled by a simple Lorentzian peak as shown in Fig. 2. The facts that fitting with Lorentzian does not fully reproduce the measured reflectivity spectra of AuFe and that for larger Fe concentrations the damping parameter $\gamma_{L}$ of the band increases imply a more complicated line shape, connected with the real complex structure of the material, such as the distribution of cluster sizes and shapes, their possible overlapping, etc. From the plasma frequency of conducting electrons in gold $\nu_{p}(\mathrm{Au}) \approx 8.5 \times 10^{3} \mathrm{~cm}^{-1}$ and in AuFe $(x=6$ at. \%) $\nu_{p}(\mathrm{AuFe}) \approx 6 \times 10^{3} \mathrm{~cm}^{-1}$, we estimate that approximately $50 \%$ of the Drude spectral weight $f_{D}$ is transferred to this band, i.e., about $50 \%$ of itinerant electrons are effectively localized within the clusters. The remaining free electrons provide a metallic dc conductivity; hence no $\rho_{\mathrm{dc}}(T) \propto T^{1 / 4}$ dependence is observed in AuFe, ${ }^{5}$ as expected for conduction systems with partially localized carriers. ${ }^{25}$

The main changes in the optical properties of AuFe caused by cluster formation start to take place at concentrations between $x=4$ and 6 at. \%; already at $x=6$ at. \% the discrepancy between the dc and terahertz conductivities is clearly pronounced and the infrared peak is developed. This agrees well with the superparamagnetic behavior observed in AuFe with rather low $\mathrm{Fe}$ concentrations of about 5 at. $\% .^{21}$

The development of a microscopic model for the optical response of highly conducting system with magnetic clusters or domains could yield further information about the real magnetic structure of the concentrated AuFe alloys on the basis of the optical conductivity spectra. Such model could give insight into the microscopic dynamics of spins and charges also in other magnetically inhomogeneous conductors, such as manganites, ${ }^{27}$ ferromagnetic semiconductors, ${ }^{28}$ intergrain magnetoresistance materials, ${ }^{29}$ ferromagnet/ superconductor layer stacks, ${ }^{30}$ materials with spin-polarized transport, ${ }^{23}$ ferromagnetic oxides, ${ }^{24}$ etc.

\section{CONCLUSIONS}

We have performed room-temperature measurements of the optical conductivity of $\mathrm{Au}_{1-x} \mathrm{Fe}_{x}$ system by varying the Fe content up to 22 at. $\%$ and covering a broad frequency range from $10 \mathrm{~cm}^{-1}$ to $3.3 \times 10^{4} \mathrm{~cm}^{-1}$. For Fe concentrations below 4 at. \% the magnetic contribution to the ac (at frequency $10 \mathrm{~cm}^{-1}$ ) and the dc resistivity of AuFe are practically equal while for higher concentrations the ac resistivity is significantly smaller. In addition, a broad absorption peak is observed at frequency of $1000-3000 \mathrm{~cm}^{-1}$ for concentrations $6-22$ at. \%. Both observations are associated with ferromagnetic clusters that cause partial localization of conduction electrons.

\section{ACKNOWLEDGMENTS}

We acknowledge discussions with A. A. Mukhin, J. A. Mydosh, and A. K. Zvezdin. This work was supported by the Deutsche Forschungsgemeinschaft (DFG) and the Russian Foundation for Basic Research under Grant No. N06-0216010-a.
${ }^{1}$ J. A. Mydosh, Spin Glasses: An Experimental Introduction (Taylor and Francis, London, 1993).

${ }^{2}$ Spin Glasses and Random Fields, edited by A. P. Young (World Scientific, Singapore, 1997).

${ }^{3}$ P. J. Ford, T. E. Whall, and J. W. Loram, Phys. Rev. B 2, 1547 (1970).

${ }^{4}$ V. Cannella and J. A. Mydosh, Phys. Rev. B 6, 4220 (1972).

${ }^{5}$ J. A. Mydosh, P. J. Ford, M. P. Kawatra, and T. E. Whall, Phys. Rev. B 10, 2845 (1974).

${ }^{6}$ P. J. Ford and J. A. Mydosh, Phys. Rev. B 14, 2057 (1976).

${ }^{7}$ I. A. Campbell, P. J. Ford, and A. Hamzic, Phys. Rev. B 26, 5195 (1982).

${ }^{8}$ M. Dressel and G. Grüner, Electrodynamics of Solids (Cambridge University Press, Cambridge, England, 2002).

${ }^{9}$ B. Gorshunov, A. S. Prokhorov, S. Kaiser, D. Faltermeier, S. Yasin, M. Dumm, N. Drichko, E. S. Zhukova, I. E. Spektor, S. Vongtragool, M. B. S. Hesselberth, J. Aarts, G. J. Neuwenhuys, and M. Dressel, Europhys. Lett. 76, 938 (2006); A. S.
Prokhorov, V. B. Anzin, D. A. Vitukhnovski, E. S. Zhukova, I. E. Spektor, B. P. Gorshunov, S. Vongtragool, M. B. S. Hesselberth, J. Aarts, G. J. Nieuwenhuys, M. Dumm, D. Faltermeier, S. Kaiser, S. Yasin, and M. Dressel, JETP 103, 887 (2006).

${ }^{10}$ E. Morenzoni, H. Luetkens, T. Prokscha, A. Suter, S. Vongtragool, F. Galli, M. B. S. Hesselberth, N. Garifianov, and R. Khasanov, Phys. Rev. Lett. 100, 147205 (2008).

${ }^{11}$ A. A. Volkov, Yu. G. Goncharov, G. V. Kozlov, S. P. Lebedev, and A. M. Prokhorov, Infrared Phys. 25, 369 (1985); A. A. Volkov, G. V. Kozlov, and A. M. Prokhorov, ibid. 29, 747 (1989); G. Kozlov and A. Volkov, in Millimeter and Submillimeter Wave Spectroscopy of Solids, edited by G. Grüner (Springer, Berlin, 1998), p. 51; B. Gorshunov, A. Volkov, I. Spektor, A. Prokhorov, A. Mukhin, M. Dressel, S. Uchida, and A. Loidl, Int. J. Infrared Millim. Waves 26, 1217 (2005).

${ }^{12}$ The procedure is described in details in M. Dressel, N. Kasper, K. Petukhov, D. N. Peligrad, B. Gorshunov, M. Jourdan, M. Huth, and H. Adrian, Phys. Rev. B 66, 035110 (2002); A. V. 
Pronin, B. P. Gorshunov, A. A. Volkov, G. V. Kozlov, N. P. Shabanova, S. I. Krasnosvobodtsev, V. S. Nozdrin, and E. V. Pechen, JETP 82, 790 (1996); A. V. Pronin, M. Dressel, A. Pimenov, A. Loidl, I. V. Roshchin, and L. H. Greene, Phys. Rev. B 57, 14416 (1998).

${ }^{13}$ Handbook of Optical Constants of Solids, edited by E. D. Palik (Academic, Orlando, 1985), Vol. I.

${ }^{14}$ R. C. Sundahl, T. Chen, J. M. Sivertsen, and Y. Sato, J. Appl. Phys. 37, 1024 (1966).

${ }^{15}$ B. R. Coles, in Amorphous Magnetism, edited by H. O. Hooper and A. M. deGraaf (Plenum, New York, 1973).

${ }^{16}$ B. H. Verbeek and J. A. Mydosh, J. Phys. F: Met. Phys. 8, L109 (1978).

${ }^{17}$ B. V. B. Sarkissian, J. Phys. F: Met. Phys. 11, 2191 (1981).

${ }^{18}$ Y. Nakai, M. Sakuma, and N. Kanitomi, J. Phys. Soc. Jpn. 56, 301 (1987)

${ }^{19}$ J. Xu, B. J. Hickey, M. A. Howson, D. Greig, R. Cochrane, S. Mahon, C. Achilleos, and N. Wiser, Phys. Rev. B 56, 14602 (1997).
${ }^{20}$ U. Larsen, Phys. Rev. B 18, 5014 (1978).

${ }^{21}$ J. Crangle and W. R. Scott, J. Appl. Phys. 36, 921 (1965).

${ }^{22}$ J. A. Mydosh, Magnetism and Magnetic Materials, AIP Conf. Proc. No. 24 (AIP, New York, 1975), p. 131.

${ }^{23}$ C. H. Marrows, Adv. Phys. 54, 585 (2005).

${ }^{24}$ M. Ziese, Rep. Prog. Phys. 65, 143 (2002).

${ }^{25}$ N. F. Mott and E. A. Davis, Electronic Processes in Noncrystalline Solids, 2nd ed. (Clarendon, Oxford, 1979).

${ }^{26}$ S. V. Vonsovskii, Magnetism (Wiley, New York, 1974).

${ }^{27}$ Z. Yang, S. Tan, and Y. Zhang, Phys. Rev. B 64, 024401 (2001); X. Liu, H. Zhu, and Y. Zhang, ibid. 65, 024412 (2001); J. Wu and C. Leighton, ibid. 67, 174408 (2003).

${ }^{28}$ M. Yamanouchi, D. Chiba, F. Matsukura, and H. Ohno, Nature (London) 428, 539 (2004).

${ }^{29} \mathrm{~S}$. Li and M. Greenblatt, J. Alloys Compd. 338, 121 (2002).

${ }^{30}$ Z. Yang, M. Lange, A. Volodin, R. Szymczak, and V. V. Moschalkov, Nature Mater. 3, 793 (2004). 